\title{
Synergistic mechanism of basic medical insurance and public health policy under major public crisis
}

Wei He

Hubei University

Huan liu ( $\square$ zcliuhuan@126.com )

Zhejiang University of Finance and Economics https://orcid.org/0000-0001-8772-1545

Tiantian Hu

Wuhan University

Research

Keywords: major public health, health benefits, basic medical insurance, policy, coordination mechanism

Posted Date: September 21st, 2020

DOI: https://doi.org/10.21203/rs.3.rs-76722/v1

License: (1) This work is licensed under a Creative Commons Attribution 4.0 International License.

Read Full License 


\title{
Synergistic mechanism of basic medical insurance and public health policy under major public crisis
}

\begin{abstract}
:
Background: The coordinated development of the basic medical insurance system and public health is not only related to the structural soundness of the entire country's medical and health system, but also related to a country's initiative in responding to major public health events.

Methods: This article attempts to endogenously implement health care policies in the health production function, and from the perspective of health benefits, attempts to build a theoretical analysis of framework for the coordinated development of basic medical insurance and public health, revealing the benignly interactive circulation mechanism of the health care system and improving the health benefits of individuals and society realistic path.

Results: In the field of medical and health services, the establishment of a benign medical and health investment system can promote the improvement of the level of health output and the improvement of the health effectiveness of individuals and society, and thus promote the increase of social and individual health and welfare levels; and promote a virtuous cycle of overall healthy reproduction.
\end{abstract}

Conclusions: Under the synergy mechanism, the basic medical insurance policy and public health policy during the new coronary pneumonia should meet the synergy standards. Scientific and reasonable medical and health service policies should not only consider the treatment of new coronary pneumonia patients and group prevention and control, but also pay attention to the coordination of basic medical insurance policies and public health policies, and the coordination of health production and health benefits. The core of policy coordination is to protect both patients with new coronary pneumonia and the general medical and health services of residents.

Keywords: major public health; health benefits; basic medical insurance; policy; coordination mechanism

\section{Background}

With the implementation of the employee's basic medical insurance system in 1998, China's urban 
and rural residents' basic medical insurance has been gradually developed. As of the end of 2019, the number of people participating in China's new rural cooperative medical insurance, urban and rural residents' basic medical insurance and employee medical insurance has exceeded 1.354 billion, and the coverage of insurance coverage is stable over 95\%, and as early as 2011, China established the world's largest medical security network. At the same time, in 2015, the General Office of the State Council issued the "Outline of National Medical and Health Service System Planning (2015-2020)". The document clearly proposes the goal of promoting the optimal allocation of medical and health resources, with a complete system, clear division of labor, complementary functions and close cooperation is at the core, and it is in line with the needs of health and oriented to the national economy and social development. An integrated medical and health service system is established to provide a reliable medical and health service system that basically covers urban and rural residents by 2020 and provides reliable health quality improvement and guarantee of medical and health resources. The special nature of medical and health services has led to the inevitable market failure of the medical and health market, which requires the government to intervene to improve efficiency and ensure fairness. Medical and health services can improve the health of the whole society and the level of individual and social health benefits by affecting the health of individuals. To improve the medical and health service system is to enhance the systematization, integrity and synergy of basic medical insurance and public health policies within the framework of existing medical and health policies.

From the point of view of the establishment of the system, basic medical insurance and public health policies have their own functional positioning, and do not have a basic synergy. As a basic medical insurance system with fairness as the starting point, it is a basic human rights system that safeguards the right to individual health. Unlike other social insurances, the core value of the basic medical insurance system is the pursuit of "equality". [1] The current research core of China's basic medical insurance focuses on integrated development [2-3], universal medical insurance [4-6] and payment method reform [7-8]. From the perspective of system practice, the basic medical insurance system for urban residents in China has undergone changes from labor insurance, publicly funded medical care to the basic medical insurance system for employees with "integrated accounts" as the core; [9] urban and rural residents' medical insurance has experienced a change from family security to the social insurance model; [10-11] The development and improvement of the new medical reform since 2011 to the universal medical insurance [12]. However, from the perspective of the overall system practice and research and development logic, the basic medical insurance reform has begun to focus on the medical cost sharing mechanism from the early stage to universal coverage. The four systems of medical services, medical security, and drug supply security, that is, the new round of medical reform, focus on the correlation between the four systems, and the core is to strengthen the requirements for collaborative development. 
Among them, policy tools are the key path to achieve coordinated development of various systems. From the perspective of policy tools, health care is essentially a reform of a series of policy tool selection and combination. [13] As one of the important tools of the health care system reform, public health policy has experienced three important stages before and after the founding of the people's Republic of China. The first stage of development before the founding of the People's Republic of China; the second stage of development after the founding of the People's Republic of China before the reform and opening up; after the reform and opening up, especially the phase of policy adjustment and transformation from SARS in 2003 to "New Coronary Pneumonia" in 2020. But what can be found is that in different historical development periods, according to different development situations such as the top-level institutional environment, values, institutional systems, and mechanism goals, it is the basis for the adjustment and reform of the public health policy framework. However, the research on the adjustment process shows that due to deviations in the legitimacy and legitimacy of public health policies, resource allocation for policy implementation, execution capacity building of grassroots units, and policy enforcement regulations, etc., resulting in significant deviations in actual implementation . [14] The effectiveness of public health policy is the key to promoting the reform of the basic medical insurance system, and it is also an important supporting point for comprehensively promoting the health level of the whole people. In response to the problems of existing public health policies, scholars have emphasized the importance of the two dimensions of "value consensus level" and "government status preference", and policy changes have universal objectivity. The specific impact path is through policy The process of maintenance, policy continuity, policy superposition and policy substitution is achieved. [15] In the process of change, the "value consensus level" will become the internal dynamic of public health policy, and the "government status preference" will directly affect the type and value orientation of public health policy.

The shortcomings of the coordination between the public health policy and the basic medical insurance system were magnified during the "new coronary pneumonia" in 2020. For example, the limitation of medical and health service resources, and the long-term government's emphasis on treatment and prevention in the field of public health investment have resulted in the fact that preventive medicine and clinical medicine are increasingly disconnected in actual medical services and that the medical system and disease control system are independent of each other. Such problems have caused obvious deficiencies in the close integration and coordinated response of medical research, disease treatment, and epidemic prevention and control under major public health events. [16] On the one hand, in terms of medical service resources, combined with the current international "Public Health 3.0" model [17] and the "One Health" concept of achieving overall health [18], strengthening public health resource integration is to play a coordinated and effective The key to sexuality, such as the coordination of medical service personnel training. On the other hand, in terms of public health financial investment, the fundamental purpose of health investment is to improve the health of residents. Early studies have shown that public health 
investment has no impact on health. [19-21] It's found that there is a strong and significant relationship between the two, because compared to horizontal cross-country studies, from a country's vertical development perspective, public health financial investment has a more significant effect on national health . [22-23] Among them, scholars based on the research results of developed countries show that there are inconsistent conclusions on the impact of public health fiscal investment on the health of residents [24-25]; based on the comprehensive research of developed countries and developing countries. It is believed by most scholars that public health financial investment has no significant effect on residents' health; [26-29] studies based on underdeveloped countries have found that public health financial investment significantly affects residents' health [30-32]. Chen et al.(2018) based on the research on the proportion of China's public health fiscal investment in GDP but found that there are obvious threshold effects, the thresholds are located at $1.9 \%$ and $6.62 \%$, and when the proportion exceeds $1.9 \%$, the scale effect of public health investment begins to manifest, the impact on the health outcome indicators was significantly enhanced; when the proportion exceeded $6.62 \%$, the results were no longer significant [33].

Combining with the current special period of "New Coronary Pneumonia", with regard to the coordinated development and research status of basic medical security and public health policy, it can be found The system design of the unity began to emphasize the importance of the relationship between the two. However in practice and academic research, scholars did not analyze the synergy between the two as an important focus. For example, scholars pay more attention to integration, universal medical insurance and payment method reform in basic medical insurance, and focus on two-dimensional research on "value consensus level" and "government status preference" in public health policy; in terms of synergy between the two, scholars have uncovered the effect of policies on residents' health and financial security from a synergistic perspective, emphasizing the impact of public health policy on financial input and neglecting to discuss medical and health services on residents' health security from a holistic perspective. This also raises new difficulties for the policy objectives. What kind of medical and health policy should be formulated to improve the national health security level? How to realize the synergy between basic medical insurance policy and public health policy to promote the most effective role of the system to ensure the national health level in major public health events? Based on the above two points, the article will combine the actual and existing research at home and abroad, from the perspective of building a health and welfare production function, to explore the internal mechanism of the coordinated development of basic medical insurance policies and public health policies and the key to a benign interactive development in order to promote national health protection System development and promotion of the optimization of medical and health policies in response to major public health practices. Compared with the existing research, the main innovation contribution of the article are: first, to construct the health and welfare production function, revealing the formation mechanism of a virtuous circle of health output brought about by 
the cooperation of basic medical insurance and public health policies under the government's financial medical and health investment. Second, starting from the corresponding policy categories of basic medical insurance policy and public health policy, the policy analyzes the internal logic of the synergy between the two, and through the two aspects of medical service and funding in medical and health, explores the realization path of basic medical insurance policy and public health policy under major public health events.

\section{Methods}

Expansion of Health Production Function and Health Benefits under Medical and Health Policy

The health demand model was first proposed by Grossman (1972), [34] and the health production function as the core of the supply model is derived from the health demand model, and the health production function can be divided into macro and micro parts, which are related to each other. Among them, the micro-health production function emphasizes the relationship between family or individual health input or medical health input and individual health output through macro-policy intervention; [35-37] The macro-health production function is based on economic factors, government health finance From a macro perspective of expenditure and medical insurance, [19] [38] consider the overall output effect of national health. The article will also introduce basic medical insurance and public health policies from a macro perspective, and conduct a theoretical analysis of the macro health production function.

\subsection{Health Production Function Expansion}

Filmer et al built a macro health production function model based on Grossman's health demand model. Health needs are formed by the association of health with related factors that help improve or enhance health. The factors of output and health input constitute the core of the health production function. Due to the deviation of relevant assumptions in the micro-field, there is an estimation error in the use of a completely competitive market model to analyze the input and output of healthcare policies and to explain the endogenous factors. Health economists use the general production function theory, combined with health characteristics, to propose that in the process of maintaining or improving health, the input and output of medical and health resources also constitute the basic health production function. That is, the general health production function can be expressed as $H=F(S, Y, E, P, Z)$, it is the national health level at a certain point in time, and $S$ represents the input of social factor variables; the $Y$ represents input of economic variable factors; the $E$ represents input of education variables; $P$ represents investment in healthcare policies, $Z$ means the health investment made by the social enterprise; $\alpha$ means the initial health output (initial health level) without any external factor intervention. The result of converting it into Cobb Douglas production function form is: 


$$
H=\alpha \Pi S_{i}^{a_{i}} \Pi Y_{j}^{b_{j}} \Pi E_{k}^{c_{k}} \Pi P_{g}^{d_{g}} \Pi Z_{f}^{e_{f}}
$$

The logarithm of both sides can be obtained:

$$
\ln H=\ln \alpha+\sum a_{i} \ln S_{i}+\sum b_{j} Y_{j}+\sum c_{k} E_{k}+\sum d_{g} P_{g}+\sum e_{f} Z_{f}
$$

When there are no major changes in other factors, and only considering the coordination of medical and health policies as the core of consideration, equation (2) can be adjusted accordingly, and mainly reflects the coordination of policies. Here, the policy is divided into two parts: public health and basic medical insurance, and it is assumed that there are three functional states of division, coincidence, or coordination. Among them, policy coordination is to pursue the optimal state of health output. Therefore, formula (2) can be adjusted according to the above assumptions to obtain formula (3), namely:

$$
\ln H=\ln \alpha+\sum a_{i} \ln S_{i}+\sum b_{j} Y_{j}+\sum c_{k} E_{k}+\sum d_{g}\left(P_{b}^{e}+P_{p}^{e}\right)+\sum e_{f} Z_{f}
$$

Equation (3) $P_{b}^{e}$ indicates the expected effect of the basic medical insurance policy or system, and $P_{p}^{e}$ indicates the expected effect of the public health policy or system. In the case of coordinated medical and health policies, $P_{b}+P_{p}=P_{b}^{e}+P_{p}^{e}$, but due to various problems such as division or overlap in the actual system or policy operation, the overall health output level of the medical and health policies is lower than the expected system design, that is, it exists $P_{b}+P_{p}<P_{b}^{e}+P_{p}^{e}$. Here, public health and basic medical insurance are introduced into the health production function at the same time, in order to theoretically investigate the impact of different system integration methods on national health output.

\subsection{Medical and Health Policy and Health Welfare Realization Mechanism}

Medical and health investment is an important part of medical and health policy, which includes two parts: basic medical insurance and public health. The basic medical insurance guarantees the medical burden of residents at the level of reimbursement through the scope of protection. Specific restraint mechanisms include demand-side constraints (such as minimum-paying-line, co-payment ratio, and capping-line demand-side constraints) and supplier constraints (such as total budget, DRGS and other supplier restraint mechanisms). Public health takes the basic public health services and medical service guarantees as its core, and exerts its role in regulating health. Among them, basic public health services use financial burden as the main tool, and through the basic disease prevention, infectious disease prevention and other policies The role of ensuring the health of residents; medical and health services are based on service supply, to ensure that residents have sufficient medical service resources after illness. Under the function of health 
production, medical and health investment realizes the overall health output of residents under the combined effects of basic medical insurance and public health, that is, disease prevention and diagnosis and treatment cost sharing mechanism. At the individual level, it manifests itself as self-utility, such as healthy human capital investment and self-care investment. From the perspective of the entire society, the end result of a benign medical and health policy is the improvement of health and welfare.

Theoretically, health benefits can be divided into social welfare and individual welfare. From a social point of view, the medical and health investment based on a country's medical and health policies will affect the national health outcomes and distribution to a certain extent, such as "life expectancy", "disease incidence", and unequal distribution of health resources The health of all citizens has improved. From an individual point of view, the health care policy is an orderly intervention of individual health determinants in different life stages, resulting in an aggregation effect. That is to say, for individuals, policy protection has caused a reduction in disease loss, improved quality of life, and increased individual human capital And so on, to form individual health resources. In the process of reproduction, the health resources brought by individuals due to the aggregation effect will be further invested in the health production chain, and will bring a new round of social and individual health benefits, and ultimately improve the society's overall medical resource consumption reduction and individual health Quality improvement. The logical relationship of the above analysis is shown in Figure 1. In the benign health production function, the effect of medical and health policy is not a closed loop, but will output social and individual welfare, and then produce an expanded health reproduction effect .

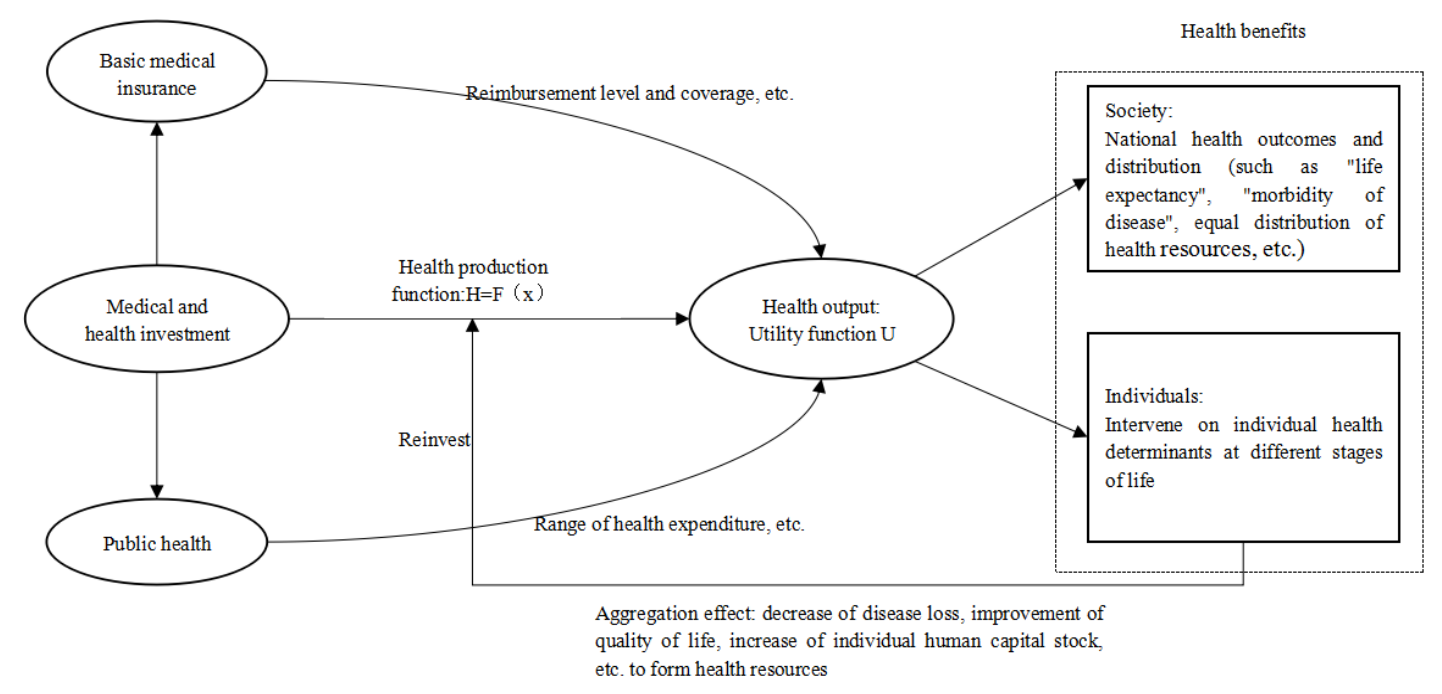

Figure 1 Relationship between medical and health policies and health benefits

\section{Results}




\section{The Benign Synergy between Basic Medical Insurance and Public Health}

The traditional research on the health production function is no distinction between public health and basic medical insurance and other macro policy synergies, so that the health welfare research conclusions focus more on single-sided analysis, lack of necessary interaction, and this phenomenon is more practical As serious. Behind this phenomenon is the combined effect of multiple factors, on the one hand is the ambiguity of health benefits, on the other hand is the complexity of the relationship between basic medical insurance and public health system. Based on existing practice and theoretical research, the article attempts to build a synergy mechanism between basic medical insurance and public health.

\subsection{The Relationship between the Two: Division, Coincidence and Collaboration}

The level of social health and welfare depends on the health resources invested, the number of healthcare workers, and the health policy. The medical and health policy has also promoted the improvement of the macro-social welfare of the national health outcomes and the healthy development of distribution through welfare policies that intervene in the health factors of individuals at different stages of life, but the premise is to act on the health production process. In order to analyze the mechanism of the synergy between the basic medical insurance and public health policies on residents' health, a theoretical analysis is needed. Based on the previous analysis, policy factors play an important role in the health production function, and after constructing the health welfare function based on the effect, it can also be found that the policy factor is a key factor in the formation of social welfare. Specific as shown in formula (4):

$$
\left\{\begin{array}{l}
H=f(S, Y, E, P, Z) \\
W=f\left(N_{i}, P\right)
\end{array}\right.
$$

It can be seen from equation (4) that, in theory, medical and health policies have the dual attributes of social welfare and individual welfare, whether it is a public health policy involved in the initial state of individual health, or a basic medical security policy after the occurrence of disease, all have a role For health output and health benefits. Basic medical insurance and public health are interdependent and work together on individuals. Without basic medical security, the excessively high medical expenses of an individual after illness will cause a higher social risk, such as "due to poverty due to illness, return to poverty due to illness"; Without public health, it is difficult to guarantee the initial health level of individuals, and the lack of necessary medical institutions to provide services after illness has created the dilemma of "difficult to see a doctor". Health welfare is not only the goal of health investment, but also depends on the support of health investment; while health investment is not only an important means of health welfare, but also cannot be separated from the support of health welfare. As previously analyzed, when there is no health benefit, health investment will not play a direct virtuous cycle (only one-way investment affects). 
On the one hand, the overall social health level is excessively dependent on government financial input; on the other hand, When major public health events occur, safeguard policies that rely too much on financial support face greater challenges, namely, the existence of funding difficulties and the inadequacy of medical service resources.

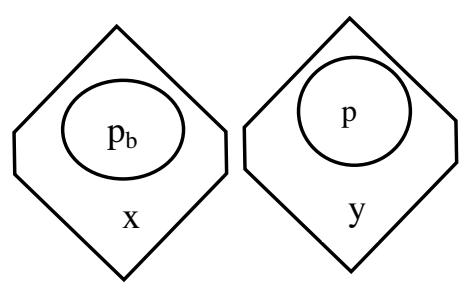

Figure 2 Policy segmentation diagram

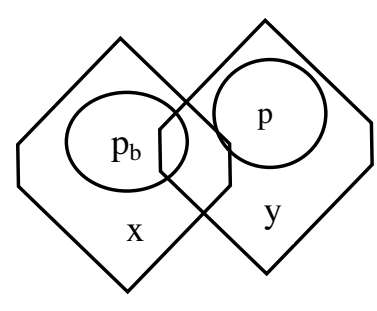

Figure 3 Policy overlap

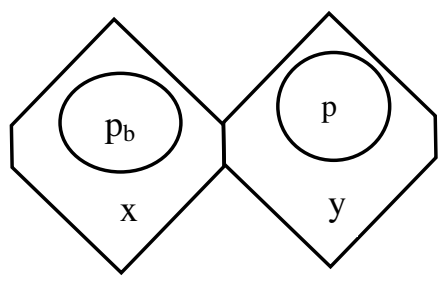

Figure 4 Policy collaboration

If a public health policy that agrees with healthy production cannot be coordinated with the basic medical insurance policy, it will cause a reduction in the overall health and welfare level of the individual or society, and may even cause serious unsustainable financial investment problems. That is to say, if basic medical insurance is separated from public health, it will cause a reduction in health production, such as a reduction in the cure rate of individual diseases and an increase in the incidence of social diseases, which will reduce the overall health and welfare level; if basic medical insurance and public health policies Crossover will result in government incapacitation, such as shirk responsibility between each other, which will result in the loss of health benefits; if the basic medical insurance and public health are fully coordinated, and the functional positioning is clear, the goals are consistent, it can effectively improve the health output and The level of individual utility, which in turn promotes an increase in the level of health and welfare of individuals and society. The basic medical insurance and public health are divided, overlapped and coordinated as shown in Figure 2, Figure 3 and Figure 4, respectively.

\subsection{Construct a Framework for the Benign Interactive Development of the Two}

Based on the previous analysis, the coordination of basic medical insurance and public health policy is the key to the health and welfare effect of medical and health policies, and at the theoretical and practical levels, how to achieve the coordinated development of the two is a core issue that needs to be urgently solved. Combining with the top-level design and level theory of the existing medical and health system, the article attempts to analyze the internal mechanism of the coordinated development of the two levels from the preventive level, the basic level, the supplementary level and the bottom level, as shown in Figure 5. 
In terms of public health, the core is to protect the health of residents and reduce the incidence of diseases. The core supply items or content are mainly primary health care and medical and health services. Primary health care is guided by disease prevention, that is, the goal of achieving fewer diseases for residents; medical and health services are oriented by basic medical and health services, and the basic goal of residents is to recover well from diseases. The two main public health projects are based on covering all urban and rural residents, relying on the security content of services and funds, and using professional public health institutions as the core of service supply to achieve the corresponding goals. Among them, strengthening health education, establishing and improving health files, reporting and handling of infectious diseases and public health emergencies are the core content of services; while government health expenditures, social health expenditures and personal health expenditures are funding guarantees and core content. As a pure public product, basic public health services must rely on government finance, and for general public health services, it is necessary to establish a social, individual, financial and other multi-party sharing mechanism to ensure that there are sufficient funds to support service development and gradually form a benign Circulation, and promote the coordinated development of the preventive layer and the basic layer under the preventive health care system.

In terms of basic medical security, its core is to compensate for the costs after the occurrence of the disease. The supply items include basic medical insurance, supplementary medical insurance, medical assistance and other multi-level systems. The protection targets are the basic medical insurance coverage group and the government financial support group. The goal of protection is to satisfy the general residents who recovers well from disease, but also to ensure that poor people can see the disease. That is, under the multi-level medical security system, the bottom goal is to achieve medical assistance to the poor, and the basic goal is To achieve general residents' medical consumption protection, the supplementary layer is to meet the higher medical consumption needs of the protected objects. Basic medical security services include basic medical services, which includes inpatient and outpatient services; fund protection is mainly for the co-payment ratio part and the top of the top line, such as the reimbursement ratio of the basic medical insurance co-payment ratio, critical illness insurance Partial reimbursement and supplementary or commercial medical insurance compensation for personal out-of-pocket expenses, so as to achieve multi-level protection at the fund level and reduce the loss of individual physical capital, human capital and social capital due to illness.

Regarding the coordination mechanism, the most direct link between public health and basic medical security is medical and health services. The former is the direct service provider, and the latter is the main funding guarantee. However, in terms of actual service supply, public health policies should not only pay attention to the supply of basic public health services, but also rationalize the layout of medical institutions and allocate resources to meet the needs of normal medical services. Because of the consistency of the two goals, if both are oriented towards 
improving the health quality of residents, they have a foundation for coordinated development. In constructing a medical service supply system, the key point is how to realize the development coordination of professional public health institutions, basic medical institutions and hospitals. Theoretically, the benign synergy between the two is the key to the improvement of the overall health and welfare of society, and is an important factor influencing the reproduction of individual health. The foundation of coordinated development is financial support, that is, clarify their respective functional positioning and determine the supply strategy based on the difference in funding sources; the key to coordination is medical and health services, through system coordination under service supply, such as basic medical and health service supply and basic medical insurance The cooperation can achieve a substantial increase in health and welfare levels.

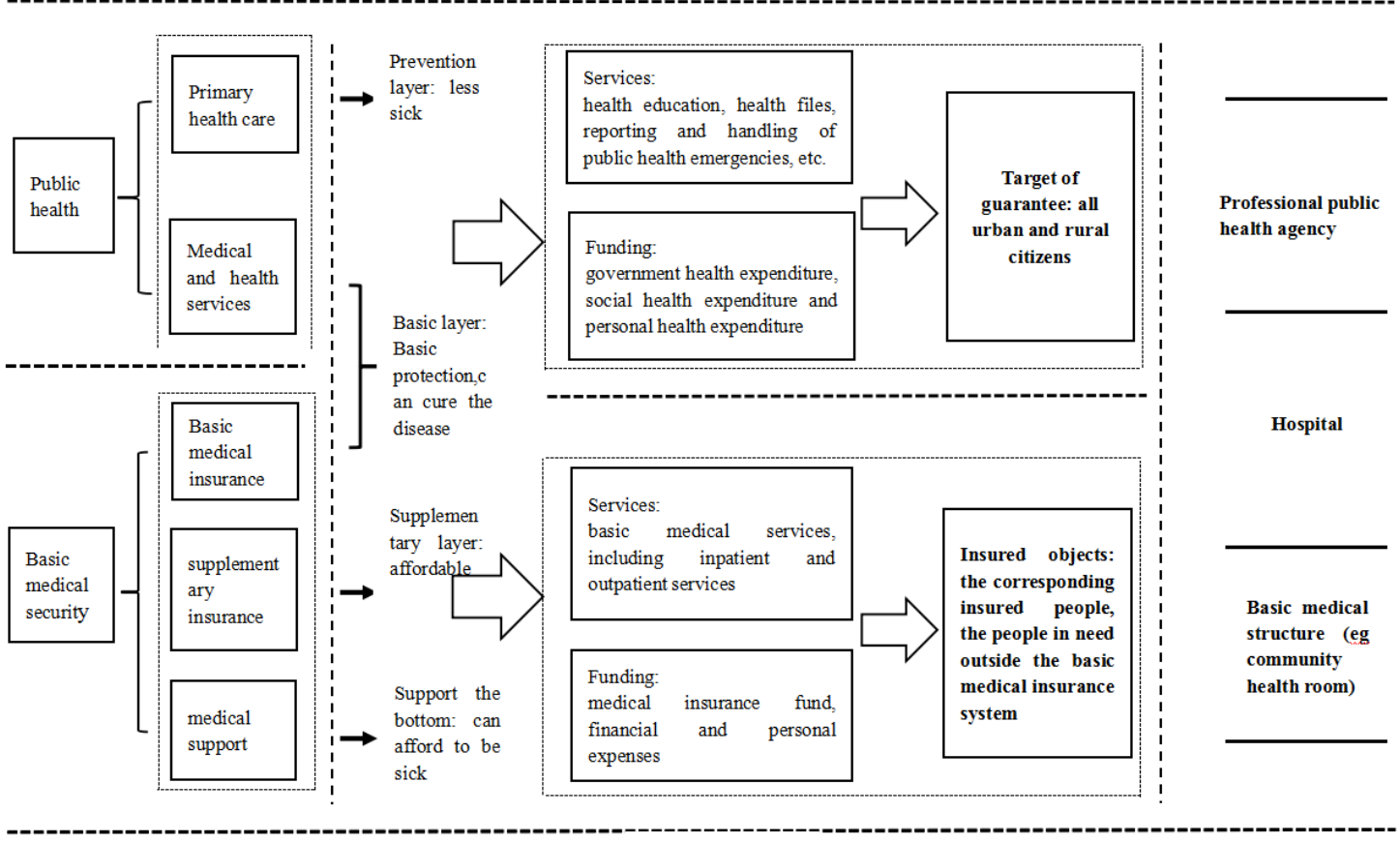

Supplier Item category $\quad$ Guarantee target $\quad$ Guarantee content $\quad$ Consumer $\quad$ Basic service system

Figure 5 Synergy mechanism between basic medical insurance and public health

The goal of the health and welfare policy is to achieve the improvement of the health of all citizens, and the focus is on the commonality and fairness of health, aiming in improving the quality of life of the citizens and reducing health vulnerability. Improving your own quality of life and reducing health vulnerability can be achieved through your own efforts, such as higher health investment for high-income groups; it can also be achieved through social policies. For the initial state of health, it is difficult to overcome the vicious circle of health or disease due to insufficient abilities The dilemma can be improved through policy interventions, which includes public health and basic medical security policies. Basic medical security policies can be divided into benefit policies (medical assistance) and incentive policies (such as supplementary medical insurance); public health policies can be divided into unitary policies and multidimensional policies, and 
unitary policies emphasize health protection in a specific area The multi-dimensional policy emphasizes the simultaneous development of multi-dimensional health services and is more in line with the requirements for achieving long-term health and welfare goals. According to the above distribution characteristics, two policy coordination paths can be explored, as shown in Figure 6.

Incentive policies in basic medical security have stronger goal consistency and mutual adaptability with multidimensional public health policies in terms of goal orientation, that is, when basic medical insurance policies are more inspiring, public health policies are more multidimensional When sex, the synergy between the two will be higher; on the contrary, when the basic medical insurance policy is more payable, and the public health policy is more unitary, the synergy between the two is very poor. The coordination degree under other combination models is also lower than that of incentive policies and multidimensional policies. Under the influence of this policy combination, the level of health output is higher and the level of health benefits is relatively better. The policy implication is that, in terms of public health supply, it is necessary not only to emphasize more welfare basic public health services, but also to appropriately develop supporting services with other dimensions, such as the establishment and layout of diversified medical service institutions, so as to achieve Multi-dimensional policy depth to meet the requirements of the basic medical insurance system; in terms of basic medical insurance, it needs to be constructed through a multi-level medical security system. Or high-income groups invest in their own health to promote the development of a multi-level medical security system and adapt to the requirements of the development of public health services.

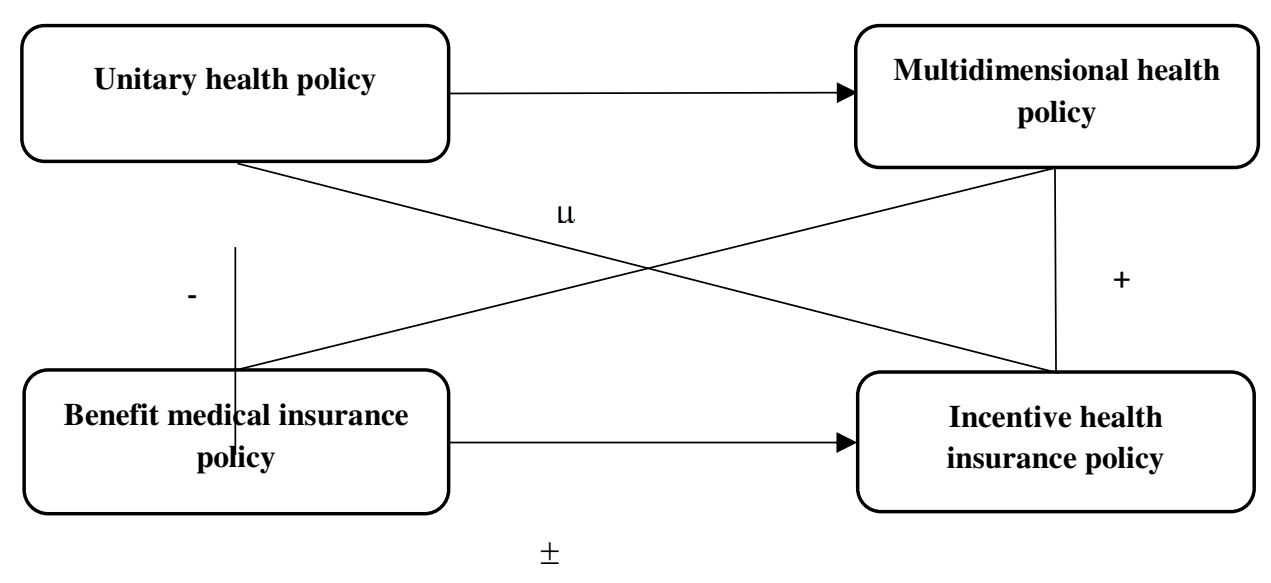


Figure 6 Synergy between different public health policies and basic medical insurance policies

\section{Discussion}

One Application: the Target Selection of Medical and Health Policy Coordination under Major Public Health Events

The new corona virus pneumonia (Corona Virus Disease 2019, COVID-19), referred to as "new corona pneumonia", the World Health Organization named "2019 coronavirus disease", refers to 2019 new coronavirus infection caused by pneumonia. During the New Coronary Pneumonia, the National Medical Insurance Bureau and the Ministry of Finance clearly put forward the "two guarantees" requirements of "ensure that patients do not affect medical treatment due to cost issues, and that medical institutions accepting treatment do not affect treatment due to payment policies. The medical insurance department requires that all diagnosed and suspected patients be treated first and then settled. After basic medical insurance, serious illness insurance, medical assistance, etc. are paid according to regulations, the personal burden will be subsidized by the finance. First of all, for confirmed cases and suspected cases, according to the "Notice on Doing Medical Security for New Coronavirus Infected Pneumonia Outbreaks" and "Supplementary Notice on New Coronary Pneumonia Medical Security" jointly issued by the National Medical Insurance Bureau and the Ministry of Finance, Comprehensive guarantees are made for medical expenses for confirmed cases, and the individual bears the financial burden; for the treatment costs of suspected cases, comprehensive guarantees are implemented by the local finance at the place of medical treatment, such as financial subsidies and appropriate subsidies from the central government. Second, after the new crown case is cured, the cost of rehabilitation is borne by the individual. They established a new designated medical service organization for coronary pneumonia and a square cabin hospital, and deploy medical personnel to support the disaster-stricken area in Hubei. From the perspective of medical and health funds and services, on the one hand, during the new coronary pneumonia, there was a problem of offside treatment of medical insurance funds; on the other hand, due to the shortage of medical institutions and medical personnel, the phenomenon of normal medical service demand was crowded out. Aiming at two problems, it is of great theoretical and practical significance to discuss the coordinated development of basic medical insurance and public health in order to improve the ability to respond to major public health events in the future.

\subsection{Medical and Health Funds Synergy}

From the perspective of health economics, under major public health events, we must first pay attention to the development of the epidemic and its losses to individuals, families and the entire society. Secondly, under the existing security system, it is necessary to re-examine the two cores of the public health system and the medical service system in the current four-in-one basic medical 
and health system in China, in order to explore the best policy to avoid or improve the response to similar major health events Combination method. Considering the potential social, economic, and health losses caused by the "New Coronary Pneumonia" epidemic, as well as direct prevention and control of economic and medical resources, the overall prevention and control of the epidemic requires high social costs. Combining theoretical and practical perspectives, it is urgent to answer two questions. The first one is whether China's current public health system is weak? And second one is, what should be the development direction of China's future public health system? Combining with the current public health system's deficiencies in responding to this major epidemic, for example, due to the split operation of the public health system and the medical service system as a whole, discussing the operating mechanism of the coordinated development of the two is to promote the two systems to better The key to seamless connection and effective coordination is also the basis for promoting the coordinated development of the health security system. The Central Committee of the Communist Party of China and the State Council issued the "Opinions on Deepening the Reform of the Medical Security System" on March 5, 2020. Its core idea is to play a strategic role in the basic medical insurance fund, and to promote the use of the medical insurance fund and public health service funds and public health services and medical services develop in concert. In terms of system design, the importance of collaboration between public health and basic medical insurance is increasing, and the measures to overpay medical insurance funds for diagnosis and treatment during the epidemic have raised a problem that must be solved for the future development of medical and health services. The burden of medical expenses under public health events urgently needs to be institutionalized.

In the basic medical and health investment system, basic medical insurance and public health each play an irreplaceable role. Under major public health incidents, the use of basic medical insurance funds for payment is the basic medical insurance policy, and the part of the self-paying part of the financial responsibility is a single public health policy. The combined effect of the two will lead to Medical and health resources cannot maximize their effectiveness. As the mechanism analysis above, the net effect of the combination of the two will be negative. The reason is that, first of all, the basic medical insurance fund pays for the treatment of infectious diseases under major public health incidents, because it is not covered by the basic medical treatment catalog, and the system does not meet the requirements; [39-40] second, because the basic medical insurance fund is offside Payment, resulting in a mechanism that caused public health fiscal expenditure to fail to effectively play its role in guaranteeing citizens' basic health and safety, and basic medical insurance emphasized the function of self-guarantee; third, the overuse of the basic medical insurance fund will inevitably cause the basic medical insurance fund As a result, the balance of income and expenditure under the pay-as-you-go financing model will be broken, and the loss of basic medical insurance foundations will cause insufficient funds for residents' normal medical services, and ultimately will still need financial support. In a major public health event, the core issue should be who bears the social risk. According to the characteristics of clustering, rapid 
transmission, and high risk of infectious diseases, it is a typical public health problem. Financial expenditure bears financial responsibility, and public health financial protection should be provided to all people who may be at risk within the territory or region, and basic medical insurance participation should not be used as a screening condition, so as to truly achieve the purpose of prevention and treatment.

In the event of a major public health event, the public health policy takes multidimensional health security as the main body, and is guided by a single financial fund guarantee, and must guarantee the publicity of financial fund expenditures to protect every individual in the country who may encounter the risk of infectious diseases to reduce the risk of reinfection that it may cause. At the same time, the basic medical security fund is still guided by incentive policies to protect the needs of all insured personnel for normal medical funds. In terms of synergy, the cost of treatment for infectious diseases (such as new coronary pneumonia) under major public health events must be borne by the public finance, and the treatment costs for other complications after infection should also be borne by the public health finance, but the The cost of comorbidity treatment should be paid by the basic medical insurance fund, so as to realize the synergy of funds between the two policies and play their respective functions.

\subsection{Collaboration of Medical and Health Services}

After the occurrence of major public health incidents, the replacement of public health financial expenditure with basic medical insurance did not cause a short-term shortfall of funds for normal medical services. The important reason is that the demand for normal medical services is crowded to a greater extent, and there is Insufficient, hidden problems.

Taking New Coronary Pneumonia as an example, there are many cases of death due to new Coronary Pneumonia in severely affected areas. These cases are crowded out due to normal medical services, which makes it difficult to meet the needs of normal medical services and is forced to give up treatment. In fact, this kind of people should also be protected under major public health incidents. However, due to the imperfection of the overall medical and health system, the strategies to deal with major public health emergencies are insufficient, resulting in shortages of medical and health resources. Therefore, based on this reality, combined with the foregoing analysis of the synergy mechanism, under major public health events, not only must there be synergy for funds, but also for medical and health services. In the early stage of new coronary pneumonia, due to lack of medical service resources, the central government deployed medical personnel to support Hubei; the lack of medical institutions, the establishment of a corresponding "square cabin" hospital to grade management and treatment of patients. This approach has achieved very good results, and effectively alleviated the problem of medical service resources in the severely affected areas, but the situation of the general medical service demand has not been greatly improved. Therefore, based on this problem, combined with specific practice, in the future 
of the reform of the medical and health system, a corresponding mechanism can be established to achieve the coordination of the two services. For institutions, according to the principles of reasonable layout and group coverage, large indoor places can be rectified or constructed in various places. The main functions can be diversified (commercial or public) during the safety period, but the ability to reserve special public health events and establish a basic reserve . In terms of medical personnel, according to the relative shortage of medical personnel under major infectious diseases, it is necessary to strengthen the construction of the medical personnel echelon system. [16] [41] During the safe period, it can cooperate with graded diagnosis and treatment, [42-43] to meet the needs of residents' medical services; in special periods, it can play a hierarchical distribution of basic medical service resources, and mildly infected persons can be treated by general nursing staff For severely infected persons, professional and high-level medical personnel are used for diagnosis and treatment, and general medical service supply and staffing must be guaranteed. In terms of mechanism, it is necessary to ensure the synergy between the multidimensional public health policy and the incentive basic medical insurance policy, especially at the level of basic medical and health service supply, to meet the needs of special medical and health services and residents' Demand for medical services, the key to synergy between the two is the supply of medical and health services, which includes many elements such as medical institutions, medical personnel and medicines.

It can be found from the practice of various countries that along with the different stages of economic development in a country, the economic structure and the structure of health expenditure are also different. Big data shows that there is a threshold effect on economic growth and the structure of health expenditures. When a country's per capita GDP exceeds US $\$ 10,000$, the total proportion of health expenditures in GDP needs to match 6\%-10\%, and the overall structure is more biased towards prevention Rehabilitation nursing accounts for $4 \%-6 \%$ of total GDP, while medical care accounts for a relatively small proportion. From general experience, the optimal ratio of preventive rehabilitation care and medical structure index when the per capita GDP exceeds 10,000 US dollars is 3:1. From the perspective of overall system coordination, the goal orientation of an efficient and high-quality medical and health system must be considered. On the one hand, we must achieve professional division of labor and reasonable structure, such as basic medical and health layout such as family doctor contract and community care; on the other hand, it is related to mobility It is compatible with integrated features, with a dot, line, and area structure leading to construct the layout of medical and health service institutions, supporting a sound system and coordinated policies, and insisting on people-oriented, thereby improving the efficiency of rapid integration of overall medical and health resources and responding to sudden public health The effect of the event.

\section{Conclusions}

With the advancement of the reform of the medical and health system, the protection of health 
needs has become increasingly important. In the traditional society, the burden of individual medical expenses will evolve into social risks, and individual health risks will also evolve into social risks, and it is more important for individual health protection. At this time, it is difficult to cope with the self-adaptive ability of the individual, so the government needs policy intervention. Policy intervention not only needs to compensate for the cost of the disease, but also needs to establish a guarantee mechanism for the health level in the early stage of the disease and the state of health after recovery. In a country's medical and health system, health output and health benefits are realized in a variety of policy environments. Policy has become a key element of health output, and the coordination between policies has become the realization of system goals or corresponding policies The key to improving health and welfare under the target. In the field of medical and health services, the establishment of a benign medical and health investment system can promote the improvement of the level of health output and the improvement of the health effectiveness of individuals and society, and thus promote the increase of social and individual health and welfare levels; and promote a virtuous cycle of overall healthy reproduction.

Medical and health investment is not only the foundation of healthy production, but also an important prerequisite for health and welfare. The joint role of basic medical security and public health will not only promote the development of social health and welfare, but also improve the level of individual health and welfare. It has the duality of macro welfare and micro welfare effects. From the perspective of social welfare, public health policy improves the overall national life expectancy and reduces the incidence of disease through the provision of basic public health services and medical health services. The goal is fairness; from the perspective of individual welfare, basic medical insurance policies and public health policies By influencing the health factors of different life stages of an individual, the individual's health level is improved and then acted on healthy production to form a benign aggregation effect, and the goal is efficiency. Looking at the separation of health benefits, the basis for the improvement or benign interaction of individual health benefits and social health benefits is the coordinated development of basic medical security policies and public health policies, that is, from the perspective of medical and health investment, both basic medical security Moderate incentives must also have the multidimensional nature of public health policies.

Under the synergy mechanism, the basic medical insurance policy and public health policy during the new coronary pneumonia should meet the synergy standards. Scientific and reasonable medical and health service policies should not only consider the treatment of new coronary pneumonia patients and group prevention and control, but also pay attention to the coordination of basic medical insurance policies and public health policies, and the coordination of health production and health benefits. The core of policy coordination is to protect both patients with new coronary pneumonia and the general medical and health services of residents.

\section{References}


[1] $\mathrm{Hu} \mathrm{H}, \mathrm{Xu} \mathrm{Y}$, Chen Q,et al.Developing a Basic Medical Insurance Statement in Chinese Underdeveloped Areas. IRAN J PUBLIC HEALTH.2018; 47(4):489-98.

[2] Liu X, Zhang X , \& Yang X. Fraud Risk Measurement of Basic Medical Insurance for Urban and Rural Residents in China. ECON COMPUT ECON CYB.2019;53(3/2019):277-96.

[3] Huang F, \& Li G.Impact of China's Urban Employee Basic Medical Insurance on Health Care Expenditure and Health Outcomes. Nber Working Papers.2015.

[4] Zhu K, Zhang L, Yuan S, et al.Health Financing and Integration of Urban and Rural Residents' Basic Medical Insurance Systems in China. INT J EQUITY HEALTH.2017;16(1):194.

[5] Timmermans S, \& Oh H.The Continued Social Transformation of the Medical Profession. J Health Soc Behav.2010;51 Suppl(1 Suppl):S94-106.

[6] Dugani S, Veillard J, \& Evans T G.Quality Primary Health Care Will Drive the Realization of Universal Health Coverage. Can Med Assoc J.2018;190(15):453-4.

[7] Feng Y, Xiong X, Xue Q,et alThe Impact of Medical Insurance Policies on the Hospitalization Services Utilization of People with Schizophrenia: a Case Study in Changsha, China. Pak J Med Sci.2013;29(3):793-798.

[8] $\mathrm{Hu} \mathrm{H}, \mathrm{Xu} \mathrm{Y,} \mathrm{Chen} \mathrm{Q,et} \mathrm{al.Developing} \mathrm{a} \mathrm{Basic} \mathrm{Medical} \mathrm{Insurance} \mathrm{Statement} \mathrm{in} \mathrm{Chinese}$ Underdeveloped Areas.IRAN J PUBLIC HEALTH.2018;47(4):489-98.

[9] Fang H, Eggleston K, Hanson K,et al.Enhancing Financial Protection under China's Social Health Insurance to Achieve Universal Health Coverage. BMJ.2019;365:12378.

[10] Chen R, Li NX, \& Liu X.Study on the Equity of Medical Services Utilization for Elderly Enrolled in Different Basic Social Medical Insurance Systems in an Underdeveloped City of Southwest China. INT J EQUITY HEALTH.2018;17(1):54.

[11] Institute of Medical Information, Chinese Academy of Medical Sciences.15 Years of Development of New Rural Cooperative Medical Care .Beijing: Peking Union Medical College Press.2018.

[12] Dong K.Medical Insurance System Evolution in China. China Econ Rev.2009;20(4), 591-97.

[13] Meng QY,Anne M,Wang LD,et al.What Can We Learn from China's Health System Reform?BMJ.2019;365: 12349.

[14] Chen MY, Liu SY, \& Perng YH.Probe into the Development and Strategies of Implementation for Intelligent Buildings in Taiwan.Appl Mech Mater.2014;584-86.

[15] Huang Y.International Institutions and China's Health Policy. J HEALTH POLIT POLIC.2015;40(1): 41.

[16] Zhang Q, He YJ, Zhu YH,et al.The Evaluation of Online Course of Traditional Chinese Medicine for Mbbs International Students During the Covid-19 Epidemic Period.INTEGR MED RES. 2020;9(3): 100449. 
[17] Desalvo K B, O’Carroll, Patrick W, et al.Public Health 3.0: Time for an Upgrade. AM J PUBLIC HEALTH.2016;106(4):621-22.

[18] Fiona G, Adele W.Healthy People, Healthy Animals, and a Healthy Environment: One Health. BMJ.2018: $\mathrm{k} 3020$.

[19] Auster R, Leveson I, Sarachek D.The Production of Health, an Exploratory Study.J HUM RESOUR.1969;4(4):411-36.

[20] Cochrane AL, St Leger AS, Moore F.Health Service \"inputl" and Mortality \"outputl" in Developed Countries.J EPIDEMIOL COMMUN H.1978;32(3):200-205.

[21] Friedlander N L J.The Relationship between Medical Resources and Measures of Health: Some Additional Evidence. J HUM RESOUR.1980;15(2):200-218.

[22] Hadley J.Medicare Spending and Mortality Rates of the Elderly.Inquiry.1988;25(4):485-93.

[23] Pierre-Yves C, Ouellette P , Pilon C.Health Care Spending as Determinants of Health Outcomes.Health Econ.1999;8(7):627-39.

[24] Babazono A, Hillman A L.A Comparison of International Health Outcomes and Health Care Spending. Int J Technol Assess Health Care.1994;10(03):376-81.

[25] Berger M C,Messer J.Public Financing of Health Expenditures, Insurance, and Health Outcomes. APPL ECON.2002;34(17):2105-113.

[26] Nixon J, Ulmann P.The Relationship Between Health Care Expenditure and Health Outcomes. EUR J HEALTH ECON.2006;7(1):7-18.

[27] Barlow R, Bilkis V.Determinants of National Life Expectancy. CAN J DEV STUD.1999;20(1):9-29.

[28] Filmer D, Pritchett L.The Impact of Public Spending on Health: Does Money Matter?. SOC SCI MED.1999;49(10):1309-1323.

[29] Bokhari F A S, Gai Y, Gottret P.Government Health Expenditures and Health Outcomes. SSRN Elect J.2006;16(3):257-73.

[30] Bidani B, Ravallion M.Decomposing Social Indicators Using Distributional Data. Policy Res Working Paper.1995;77(1):125-39.

[31] Wang L M.Determinants of Child Mortality in LDCs: Empirical Findings from Demographic and Health Surveys. Health Policy.2003;65(3):277-99.

[32] Rajkumar AS, Swaroop V.Public Spending and Outcomes: Does Governance Matter?J DEV ECON.2008; 86(1):96-111.

[33] Chen J, Novak P, \& Goldman H.Public Health System-delivered Mental Health Preventive Care Links to Significant Reduction of Health Care Costs.POPUL HEALTH MANAG.2018;21(6), 462-68.

[34] Grossman M.On the Concept of Health Capital and the Demand for Health.J POLIT ECON.1972; 80(2):223-255. 
[35] Rosenzweig M R, Schultz T P.Estimating a Household Production Function: Heterogeneity, the Demand for Health Inputs, and Their Effects on Birth Weight.J Polit Econ.1983;91(5):723-46.

[36] Muller C.A Health Production Function for Quasi-Autarkic Agricultural Households in Rwanda. EUR J DEV RES.2001;13(1):87-105.

[37] Bichaka F, Paulos G.Estimating a Health Production Function for Sub-Saharan Africa(SSA). Appl Econ.2005;37(2):155-64.

[38] Thornton J.Estimating a Health Production Function for the US: Some New Evidence.Appl Econ.2002;34(1):59-62.

[39] Brown G T.Medical Futility in Concept, Culture, and Practice. J CLIN ETHIC.2018; 29(1):114-23.

[40] Hoffman G J, Hoffman J R, Howlett M,et al.Cjem Debate Series: \#Copayment-Medical Insurance Is for Non-routine Events. CAN J EMERG MED.2018;20(01):16-20.

[41] Huang Pei, Yi Lihua. Practice and Thinking on the Construction of Hospital Discipline System Based on Discipline Tree Cultivation Strategy . CHS J Hosp MANAGE, 2018, 34 (08): 695-698.

[42] Li X, Li Z, Liu C,et al.Evaluation of the Three-in-one Team-based Care Model on Hierarchical Diagnosis and Treatment Patterns among Patients with Diabetes: a Retrospective Cohort Study Using Xiamen's Regional Electronic Health Records. BMC HEALTH SERV RES.2017;17(1):779.

[43] Cai Q, Mi Y,Chu Z,et al.Demand Analysis and Management Suggestion: Sharing Epidemiological Data among Medical Institutions in Megacities for Epidemic Prevention and Control. J Shanghai Jiaotong Univer (SCI).2020;25(2):137-39. 
Figures

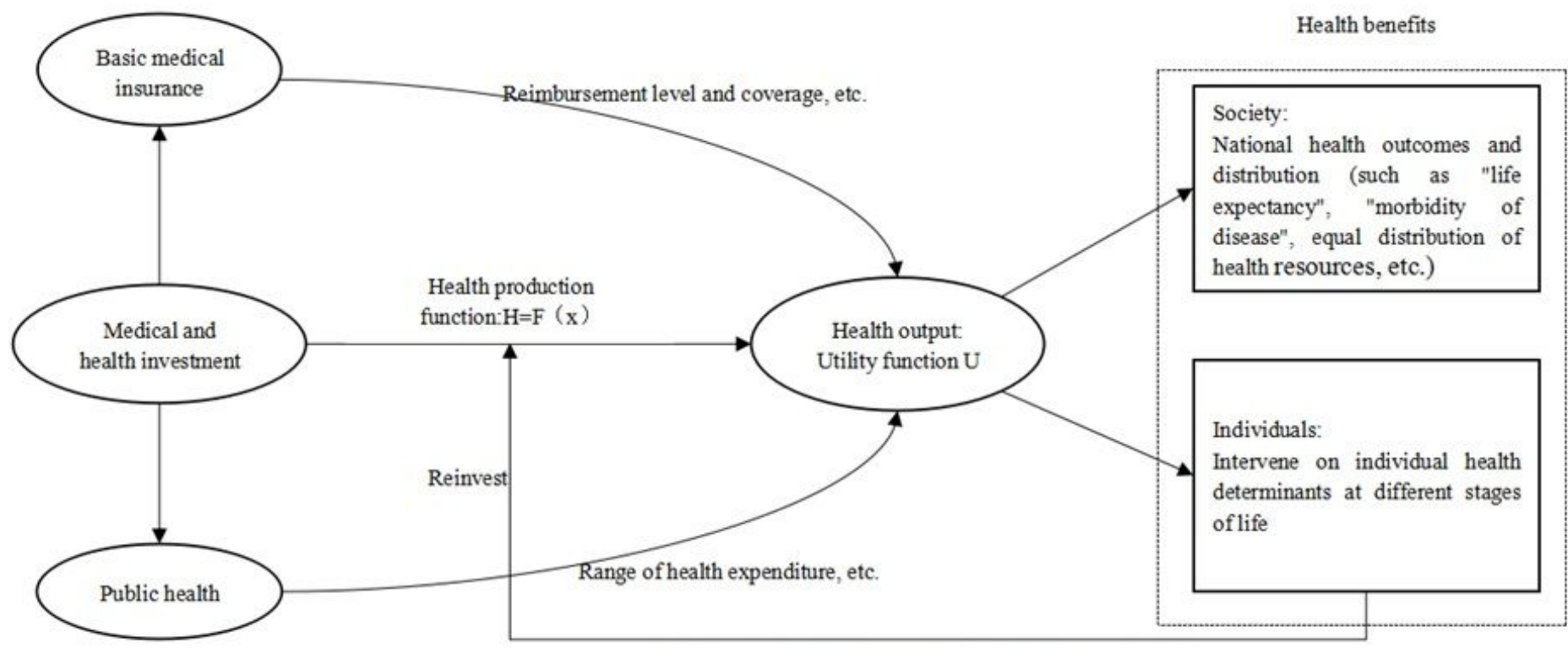

Aggregation effect: decrease of disease loss, improvement of quality of life, increase of individual human capital stock, etc. to form health resources

\section{Figure 1}

Relationship between medical and health policies and health benefits

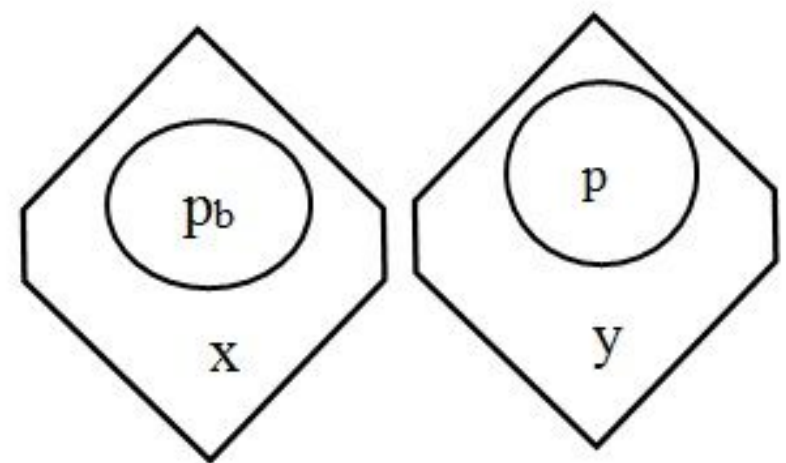

Figure 2

Policy segmentation diagram 


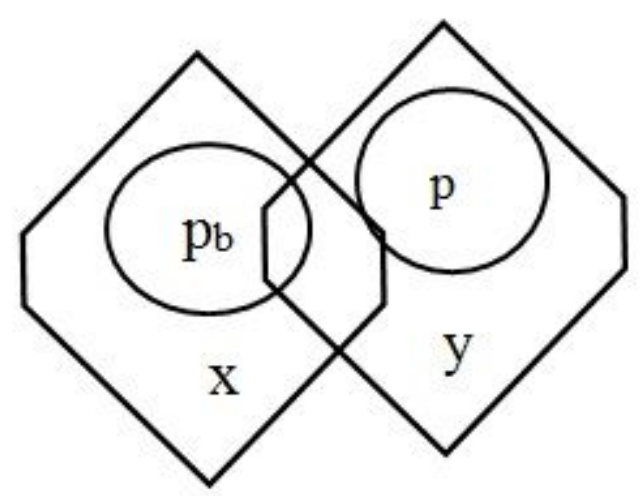

Figure 3

Policy overlap

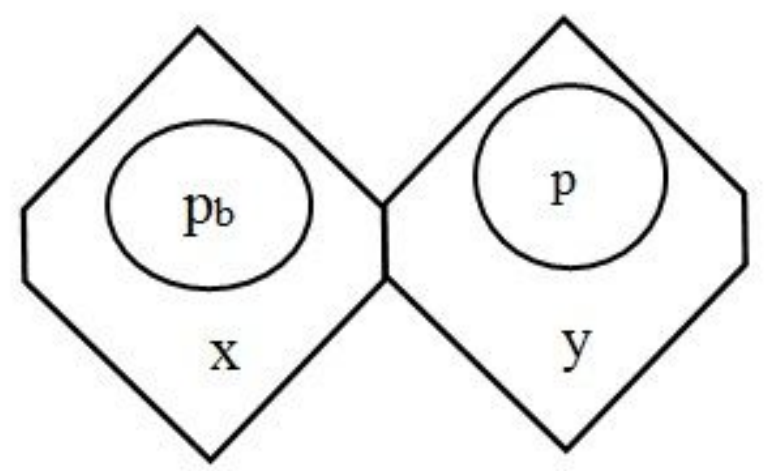

Figure 4

Policy collaboration 


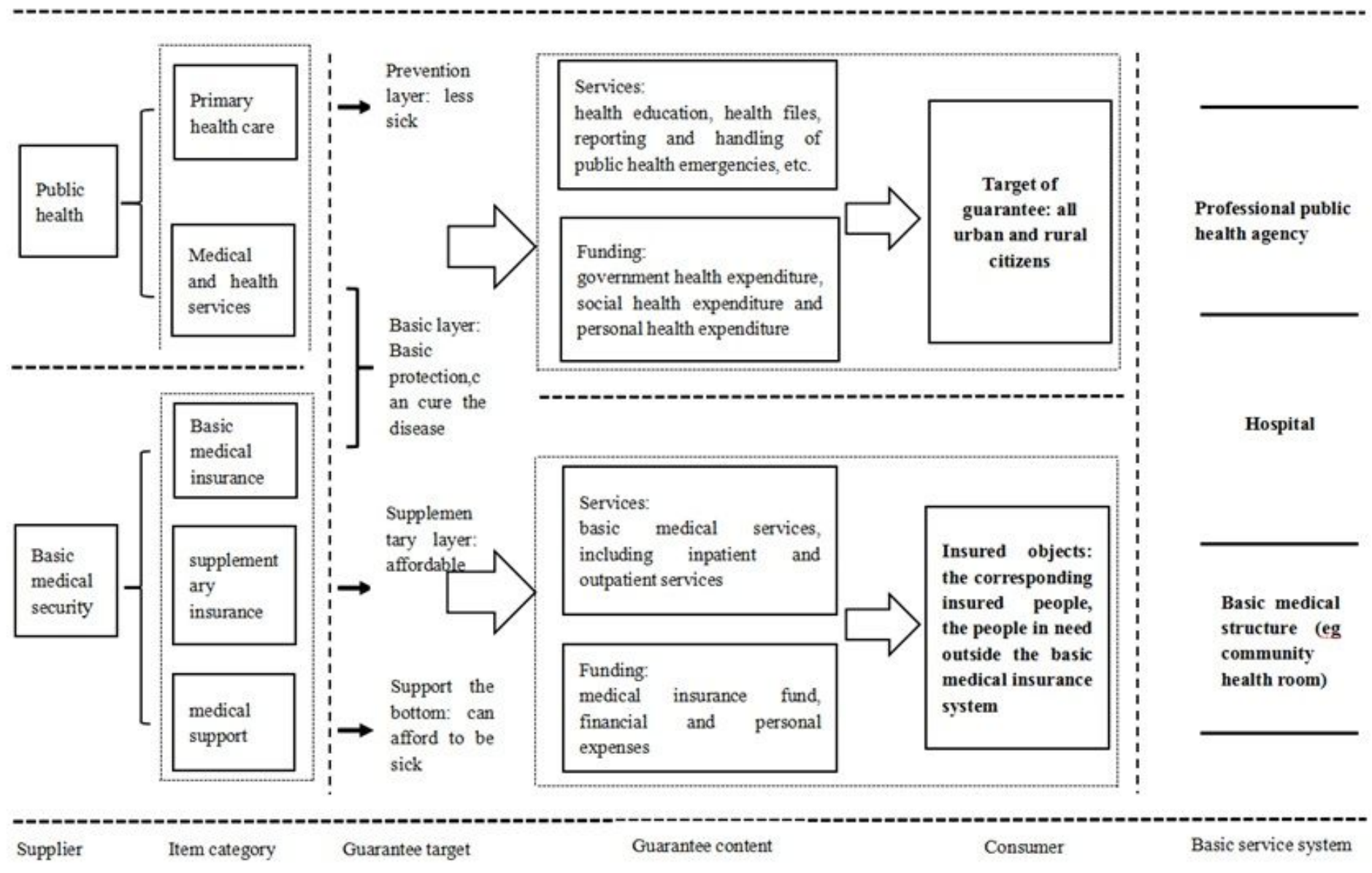

Figure 5

Synergy mechanism between basic medical insurance and public health

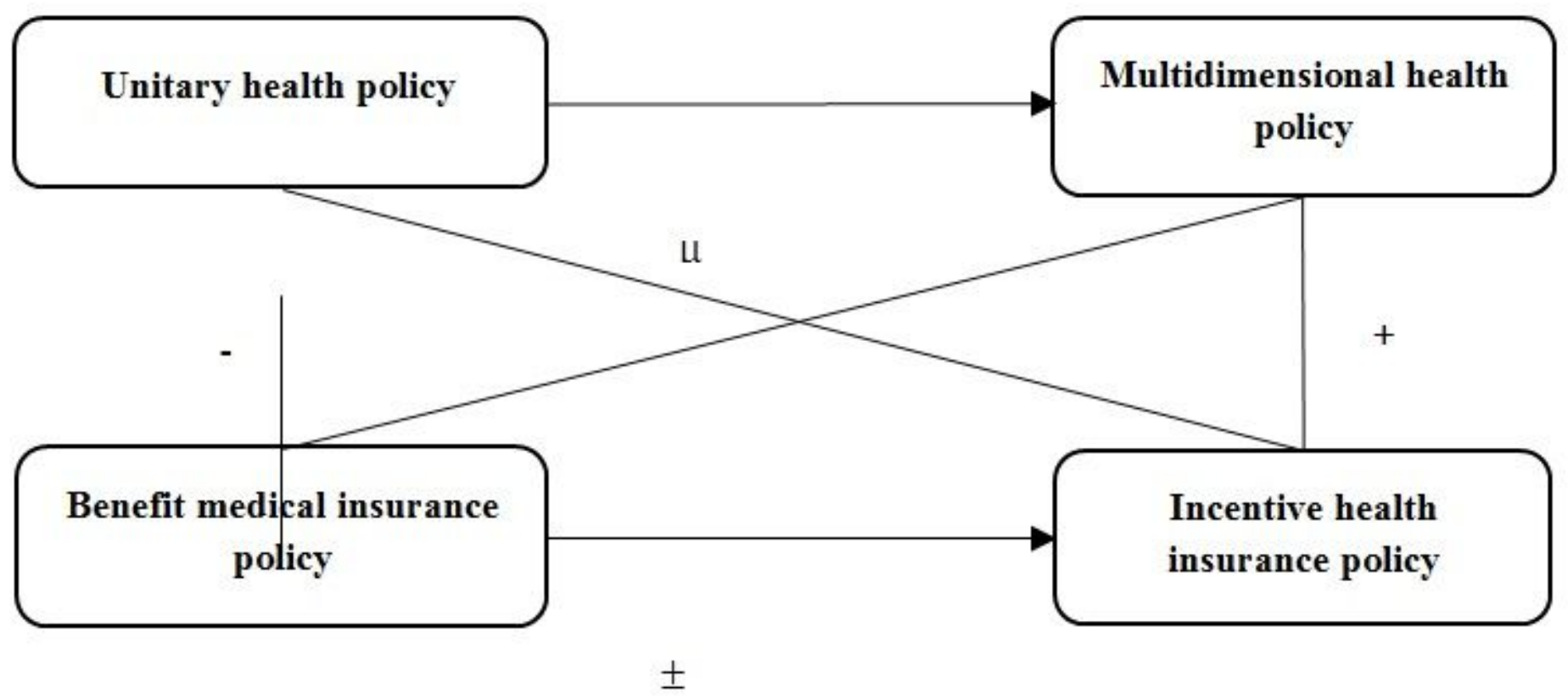

Figure 6 
Synergy between different public health policies and basic medical insurance policies 